\title{
Contextualising Case Reviews: A methodology for developing systemic safeguarding practices
}

\begin{abstract}
This paper introduces a systemic methodology for reviewing professional responses to abuse between young people. The approach, 'contextual case reviewing', draws upon constructivist structuralism to assess the extent to which safeguarding practices engage with the social and public contexts of abuse. The paper conceptually compares the methodologies of contextual case review and other serious case review methods before drawing upon findings from two studies which used the contextual case review methodology to explore the extra-familial nature of peer-on-peer abuse and the ability of child protection practices to engage with this dynamic. Thematic findings from these studies regarding the practical interpretation of 'significant harm' and 'capacity to safeguard', as well as their use within child protection assessments, are used to challenge conclusions of other case reviews which imply that child protection procedures are sufficient for safeguarding young people. Contextual case reviews suggest that safeguarding practices, and the legislation that underpins them, are culturally, procedurally and organisationally wedded to the context of the home, while insufficiently engaged with extra-familial contexts of significant harm. The application of these issues require interrogation if social work systems are to provide sufficient mechanisms for safeguarding young people and families at risk of significant harm.
\end{abstract}

Keywords: Environmental Intervention, Violence (in children, adolescents, juvenile offenders), Methods and Practice, Child protection (policy and practice), Adolescence

\section{Introduction}

Over recent decades case reviews have provided one route to scrutinise child protection systems and identify improvements required to safeguard young people. A range of methodological approaches have been developed to conduct reviews in the UK, and studies into review processes suggest that many have focused heavily on whether procedures were followed and less on systemic causes of failure (Brandon, et al., 2012; Munro, 2011). Building on these debates, this paper introduces a contextual case review (CCR) methodology, used in two studies into the nature of, and response to abuse between young people (referred to as peer-on-peer abuse for the purposes of this article). Each study used contextual case reviews to identify the public and social dynamics of peer-on-peer abuse and the engagement of services with this dimension of adolescent safeguarding. This paper draws together the findings of both studies to reflect upon the implications of the CCR approach for case review methodologies and child protection systems. Having outlined the nature of peer-on-peer abuse, the role of case reviews and their use in reviewing responses to extra-familial risk, the CCR methodology, its theoretical framework and its use in the two studies will be introduced. Thematic findings from these two studies will then be drawn upon to present the learning yielded from this methodological approach. The CCR methodology provides a lens to identify: interplay between extra-familial contexts during abusive incidents 
and the struggle of services to respond to this; the limited capacity of parents, professionals, and young people to disrupt extra-familial risk; and the inability of existing policies and procedures to address these challenges. These thematic findings provide the foundations to discuss procedural, legislative and cultural changes for safeguarding practices, which apply assessment, intervention and notions of 'capacity to safeguard' to places and professionals beyond the boundary of 'the family'. While raising universally important questions, the discussion is of particular significance for 'child protection' (as opposed to family support) approaches to social work which are modelled, to different extents, in most English speaking countries (Connolly, 2016; Parton, 2014). While acknowledging the limitations of this relatively young methodology, the CCR approach suggests the need to radically 'contextualise' child protection approaches that are limited to familial settings, in order to better safeguard young people from significant harm.

Setting the Scene: Peer-on-peer abuse, extra-familial risk and the role of serious case reviews

Over the past ten years academics, policymakers and practitioners have expressed concern about the significant minority of young people in the UK who are abused by, or who abuse, their peers (BBC, 2015; Barter, et al., 2009; Hackett, 2011; Radford, et al., 2011; Ringrose, et al., 2011). In 2012 a survey of 1,065 13-14 year olds in England found that just over half (52.5 per cent) had at least one experience of victimisation, perpetration or witnessing relationship abuse (Fox, et al., 2012) and the 2013 Crime Survey in England and Wales estimated that children aged 10-15 years old experienced 465,000 incidents of violent crime, $79 \%$ of which was perpetrated by someone aged 10-15. A third of young women have reported experiencing seuxal violence from a partner before they turn 18 (Barter et al. 2009) and two-thirds of adult survivors of child sexual abuse have said that they were abused by another young person and not an adult (Radford, et al., 2011).

In addition to demonstrating the scale of the abuse that young people experience in their peer groups and intimate relationships, analysis to date implies that peer-on-peer abuse is largely an extra-familial, contextual phenomenon. Whether it is harmful norms in peer groups (Barter, et al., 2009; Corr, et al., 2012), problematic corridor cultures and gender stereotyping in schools (Finkelhor, et al., 2009; Ringrose, et al., 2011), or exposure to harassment and victimisation on the streets (Beckett, et al., 2013; Squires \& Goldsmith, 2011), risk within extra-familial contexts can contribute to experiences of peer-on-peer abuse (Firmin, 2015; Letourneau \& Borduin, 2008).

As peer-on-peer abuse can feature physical, emotional and sexual abuse between young people, including rape, exploitation and, in rare cases, loss of life, some manifestations of peer-on-peer abuse pose a risk of significant harm to young people, and as such have been subject to case reviews.

\section{Case Reviews - an introduction}

The Children Act 2004 introduced a mechanism for local safeguarding partnerships to conduct case reviews in England and Wales. The importance of case reviews has been outlined in national child protection guidance, Working Together (2015), which promotes regular reviewing exercises to learn about, and improve, practice. Serious case reviews (SCR) are one form of such reviews, and are used when a young person has been killed or 
seriously harmed as a result of maltreatment or abuse. Child protection reviews and agency reviews/audits are conducted in less severe cases where beneficial learning could be engendered from the process.

Despite calls for systemic approaches to reviews (Munro, 2011; SCIE, 2012) most serious case reviews have adopted a management review methodology. Individual agencies submit their own management review (IMR) of a case and an overview author compiles a report following analysis of all IMRs and additional conversations with practitioners and families affected (DfE, 2016). Compared to the IMR approach, the Social Care Institute for Excellence (SCIE) has promoted a systems model of collaborative reviewing and consideration of the organisational contexts in which cases are managed to avoid reviews that are overly-concerned with procedure and process (SCIE, 2012). The CCR methodology introduced in this paper builds upon the SCIE model and in doing so adds to criticisms of case reviews that recommend improvements to policies and procedures without due regard for the context in which practice occurs.

While predominantly used in cases of maltreatment associated with young people's families, approximately a fifth of serious case reviews have considered incidents that occurred in 'community contexts' (Brandon, et al., 2012). Serious case reviews therefore have been used to learn about, and develop, responses to extra-familial risk - and as such are one tool for advancing safeguarding responses to peer-on-peer abuse.

\section{Serious case reviews and extra-familial risk}

In May 2016 the author made a request to the National Society for the Prevention of Cruelty against Children (NSCPCC) library for all serious case reviews conducted over the past ten years which featured a young person aged 10-17 and where the abuse of concern was extra-familial. The NSPCC are the only organisation to hold a catalogue of all published serious case reviews. While there are gaps in their collection (as not all serious case reviews are published) (Brandon, et al., 2012), of those available (duplications removed) 65 featured young people's experiences of extra-familial risk of which 33 were cases of peer-on-peer abuse.

Detailed analysis of these cases is featured in a separate paper (Walker and Firmin, 2017 Forthcoming) - however they are summarised here to provide an evidential context for the CCR methodology introduced in this paper. Of the 33 cases concerned with peer-on-peer abuse:

- Four involved peer-on-peer sexual exploitation

- Eight were described as involving harmful sexual behaviours

- Ten involved an incident where a young person was killed or seriously injured by a peer

- One involved the murder of a young person by a partner in a domestic abuse context

- Eleven featured suicide as a result of bullying in school $(n=10)$ and custody $(n=1)$.

Messages from these reviews are largely critical of professional responses to extra-familial risk. Many argue that social workers and the multi-agency partnerships in which they work should have utilised existing safeguarding and child protection procedures, regardless of whether that harm was located in the family - for example: 
[Following Daniel taking an overdose with peers] the Team Leader said her judgement was that his behaviour did not constitute significant harm....This was a seriously flawed judgement... it demonstrates no understanding of the fact that some teenagers become out of their parents' control and suffer or are at risk of suffering, significant harm. This is enshrined in the Children Act 1989, as a reason why a Court would consider making an order to safeguard a child. This is exactly the situation that faced these parents and the facts are absolutely clear. Daniel was only just 14; he was out of his parents' control and was suffering significant harm' (Case 47:26)

The risk assessment tool within the recent DCSF Guidance should be used by relevant agencies at an early stage where the behaviour of young people starts to escalate to anti-social behaviour and criminality. The existing processes such as the Families at Risk panel will need to incorporate this in its work. (Case 11:9)

Such conclusions suggest that the challenge with responses to these cases was the misinterpretation of child protection frameworks and not the frameworks themselves. Only one of the abstracts from the 33 peer-on-peer abuse case reviews recommended a change to the child protection system - arguing that bullying should be considered a child protection issue. Two acknowledged the challenge of peer-influence but didn't recommend any system change to address this.

Of the broader sample of 65 extra-familial case reviews, two noted the challenges faced by child protection systems in responding to extra-familial risk and a further review highlighted the need to better engage with school environments in which young people encounter harm. All three used the SCIE systemic model to collect information, rather than the IMR approach which is focused on process, and were therefore better placed to identify contextual factors. Only one other review abstract explicitly referenced 'extra-familial' risk in relation to child protection systems, despite 20 being concerned with incidents associated to schools, four associated to group influence and a further three related to peer relationships.

Importantly one review conducted in 2012, acknowledged that despite following all safeguarding processes, professionals were unable to protect young people from their peer group. The review found that processes were followed for each young person individually but were never brought together to address the peer dynamic. Responding to the needs of each individual family did little to de-escalate the collective risk that these young people encountered when they were together.

The above summary highlights a fundamental limitation with safeguarding responses to extra-familial risk and many serious case review models. Safeguarding processes are largely engaged with individual young people and families separately - even when the risk they encounter is networked, social or public. Given the contextual nature of peer-on-peer abuse, this presents a conceptual challenge for advancing safeguarding practices in general and learning from case reviews more specifically. The conclusions drawn by existing reviews largely indicate that practitioners should use existing safeguarding practices to respond to peer-on-peer abuse and that the current legislative frameworks are sufficient for achieving this. However, more thought is required to consider the small number of SCIE recommendations, and the larger number of references to peer and school influences within 
case reviews, to identify: the detailed nature of extra-familial risk that young people experience and the ability of practitioners to use existing systems in order to address this.

In order to do this the author designed CCRs. The remainder of this paper will present the CCR methodology and the lessons that have been drawn from their use in two studies that have explored the contextual nature of and response to peer-on-peer abuse. Learning yielded thus far suggests that the CCR approach has the potential to inform the development of responses to peer-on-peer abuse, case reviewing activity, and child protection policy.

\section{Contextual case reviews: a methodology and application in two studies}

CCRs were designed by the author in 2011 to explore the extra-familial nature of peer-onpeer abuse. The approach draws upon Bourdieu's theory of constructivist structuralism (Bourdieu, 1992; Powell, 2010) which recognises that an individual's behaviour is informed by, and informs, the contexts in which they spend their time. Individuals, including young people, move between contexts. Contexts may intersect, but each is demarcated by their own sphere of influence. During this process individuals embody the rules of social contexts but also possess a generative capacity to create and engage in new rules (different from those to which they have become accustomed) when they engage in alternative contexts. With reference to peer-on-peer abuse, therefore, the behaviour of young people who are abused by, or who abuse, their peers is informed by, and informs, the families, peer groups, schools and public spaces in which they spend their time.

The theoretical framework outlined above was used to design a case review methodology for examining the contextual nature of peer-on-peer abuse incidents and the extent to which this contextuality was accommodated by safeguarding responses. While the contextual characteristics of peer-on-peer abuse had been suggested by wider studies into the phenomenon (Barter, et al., 2009; Letourneau \& Borduin, 2008), the nature of social context interplay at the point of the abusive incident, and the engagement of services with this interplay, warranted further attention. A case review methodology lent itself to providing an in-depth analysis of case management and abusive behaviours, and has been utilised for this purpose in two studies into the nature of, and response to, peer-on-peer abuse.

\section{Structure of case review template}

A template was designed to facilitate CCRs. The template was introduced so that data from a range of systems compiled for any given case could be anonymised and transferred onto it in a uniform fashion. Multiple templates could be analysed to identify themes, and individual templates could be turned into vignettes to provide an anonymised account of an incident and/or a response.

The template was split into three sections.

Section (1) The Incident and the Young People Involved: this section documented the nature of the incident that was subject to case review, the escalation towards it and the behaviours involved. It also documented the individual characteristics and behaviours of the young people involved (gender, age, ethnicity, criminal offence record, periods of going missing etc.). 
Section (2) Associated Contexts: this section documented the nature of the different contexts to which featured young people were associated. Evidence of abusive behaviours, criminality, harmful norms, a lack of capacity to safeguard and protective characteristics were recorded for each context - homes, peer groups, schools and neighbourhoods.

Section (3) The Response: the engagement of services with the young people and contexts associated to the case prior to, during, and following the incident in question. This included: how the incident was identified; the immediate response from professionals; multi-agency meetings; assessments; interventions; and outcomes.

\section{Approach to data collection and analysis}

The template provided a uniform repository for data gathered for any given case review. As the template was designed for reviews commissioned by Local Safeguarding Children's Boards (the statutory functions of which in England include developing local safeguarding policy and procedures and scrutinising local safeguarding arrangements), or single statutory agencies, data collection was overseen through their governance frameworks. Initial data was drawn from the referral source and placed into the template. Following this initial capture additional records from participating agencies, concerning the young people featured in the case, were provided. Data were drawn from these records into corresponding sections of the case template.

Each young person, family, peer group, school and public space (such as the park) was given an anonymising code

The coding framework for analysing templates, and drawing conclusions, was designed to identify:

- The ways in which individual characteristics interplayed with social contexts in the pathways towards, during and following an incident of significant extra-familial harm

- The extent to which safeguarding responses engaged with the interplay between individual characteristics and social contexts as a means of addressing significant harm.

Codes were created for conducting qualitative analysis on each section of the template in this regard and then to compare them. Analysis identified trends related to where abusive behaviours/norms were associated, where the professional response was targeted and where impact was made - for example: identifying if abusive behaviours were largely located in the peer group of a young person suspected of abusing their partner, while the professional response focused on the family of the young person that they abused.

\section{Application of the in two studies}

To date this template, and the theoretical and methodological approach outlined above, has been used to analyse 18 cases across two separate studies (Table 1).

\section{INSERT TABLE 1}

Both studies sought to explore: the association between extra-familial social contexts and young people's experiences of peer-on-peer abuse; and the extent to which safeguarding practices engaged with extra-familial dynamics. They were subject to a two-stage 
independent ethical approval process at the author's university, in addition to the first study requiring ethical approval from the participating police force. Given the sensitive nature of the subject-matter both studies adhered to strict ethical parameters including agreements to only disseminate thematic learning from across cases rather than to reproduce any individual case in detail. Anonymity of research sites, practitioners and young people are further protected through the use of pseudonyms in any quotes used.

Study (1) used the template to analyse nine cases of peer-on-peer abuse (Firmin, 2015) in one urban police force area. Initial material taken from police investigations which themselves included supplementary material from children's services, schools and youth offending.

Study (2) had the same objective but formed part of a wider action research project with 11 multi-agency safeguarding partnerships to develop their local responses to peer-on-peer abuse (Firmin et al, 2016). Cases were drawn from two police forces - one metropolitan town and one rural county. On each occasion a referring agency, largely the youth offending service or a specialist service commissioned by the local authority, provided the referral material. Once this detail had been anonymised and transferred to the templates it was supplemented by information from children's social care, youth offending, youth service, education and policing records.

The findings of Study (1) raised questions about the sufficiency of largely individualised and familial systems for addressing risk that was often extra-familial (Firmin, 2017; Parton, 2014). Study (2) provided an opportunity to refine the case review template (the original section structure remained but the formatting was adapted to ease the recording process) and to consider whether the questions raised by the first study were limited to one, or spread across multiple, geographic regions.

Study (2) raised similar questions to the first, and provided further exemplifications of the ways in which existing safeguarding structures are ill-equipped to respond to peer-on-peer abuse specifically and extra-familial risk more broadly.

Before presenting the findings of this work it is important to note key limitations of the CCR approach. Firstly, CCRs are largely desk-based exercises and are therefore reliant upon the information that is recorded in assessments, intervention plans, reports, minutes and so on. It is possible that for all cases reviewed professionals who were involved had developed ideas or approaches for working with cases that were not recorded. Secondly CCRs have to date been conducted on cases where things have gone wrong and young people have been abused. It is possible that there are other cases where contextual practices were drawn upon to prevent escalating concerns and such work is not represented in the evidence documented within a CCR. As a result it is critical that evidence emerging from CCRs is analysed and discussed with reference to wider research into the nature and sufficiency of child protection practices, rather than being considered in isolation.

Findings related to the nature of peer-on-peer abuse and contextual practices that emerged from each study have been published elsewhere (Firmin, 2015; Firmin et al., 2016; Firmin, 2017). This paper draws the two studies together for the first time to present and reflect upon the CCR methodology specifically and its implications for how we understand case review conclusions and critiques of child protection systems. In this sense the findings and 
discussion below are intended to raise conceptual questions about the sufficiency of safeguarding legislative and organisational cultures that are primarily focused on families and make recommendations about the systemic change required to the better respond to peer-on-peer abuse. CCRs raise questions about the role of social workers, and the multiagency partnerships in which they operate, in shaping extra-familial contexts, and acknowledge the impact of public-spaces (and the professional management of them) on the capacity of parents to safeguard young people from significant harm.

\section{Findings: lessons from contextual case reviews}

Drawing upon findings from the two CCR studies outlined above, this section will document the challenges of:

1. Recognising risks of significant harm within extra-familial contextual interplay

2. Extra-familial risk undermining parental capacity to safeguard

3. Using child protection assessments and procedures to address these aforementioned challenges

Throughout this process the particular benefits of a CCR process for illuminating these challenges will be highlighted, before the implications of this learning for safeguarding systems are discussed.

\section{Significant harm and extra-familial contextual interplay}

As introduced earlier in this paper, young people's experiences of peer-on-peer abuse are often informed by the nature of, and their engagement with, their peer groups, schools and local neighbourhoods (both online and offline). Using CCRs across two studies into peer-onpeer abuse evidenced this contextual dynamic, and more importantly, the interplay between extra-familial contexts. What happened in young people's peer groups impacted the nature of their engagement with school, what happened in their neighbourhood informed the nature of their peer group and so on. For example, in one case a young person's exposure to violence and criminality on the streets informed the violent nature of his peer group - and his peer group in turn, and their collective desire for status and safety on the streets, prompted the young person in question to disengage from school.

In 16 out of the 18 cases reviewed, professionals struggled to articulate and/or recognise the risk of significant harm that young people encountered in extra-familial contextual interplay. For example, in the second study when a young woman was kidnapped and physical assaulted by her boyfriend and his friend, social workers assessed the nature of her family environment and concluded that there was no need for any further children's social care involvement. The relationship between this young woman's boyfriend and the peer that he offended alongside, and the influence that this had on her safety, was not subject to any form of safeguarding assessment or intervention but was clearly documented in police paperwork and youth offending reports concerned with the case. Likewise, in the first study a young woman disclosed that she had been raped by her peers in her local neighbourhood and had been sexually harassed at school. Yet during the assessment processes (which focused on her family) social workers concluded that she was not at risk of significant harm.

In the two examples above risk of significant harm escalated within peer dynamics, and then in the interplay between school, peers and the neighbourhood, but the locus of assessment 
remained the family. It is not simply that the risk was 'environmental'; it was its location in the interplay between different aspects of young people's public/social space engagement that presented challenges to professionals. The CCR methodology assists in identifying this challenge as it explicitly considers the location of the significant harm and not just its existence.

Some of the learning outlined thus far could be garnered from SCIE or other systemic approaches to case review. However, as the CCR method disaggregates 'environmental' or 'extra-familial' risk into peer group, school and neighbourhood, it is better equipped to consider the interplay between different extra-familial risks. It sharpens the focus of the reviewer on this space, and is therefore better able to consider not just the location of the incident in question, but the professionals responsible for that space (i.e. teachers in schools, community safety officers in the neighbourhood) and the weight of influence between different extra-familial contexts. For example, the influence of the social rules on the streets on a young person's behaviour can be greater than that of the rules of their school even though both are arguably environmental. Such a finding is even starker when one considers the interplay between extra and intra-familial relationships, and the impact that this has on parental capacity to safeguard.

\section{Questions of 'capacity' to safeguard}

In 15 of 18 case reviews the capacity of parents to keep their children safe was undermined by factors external to the family. Parents in nine cases contacted professionals to express concern that they were losing control of their children prior to the incident under review:

Sean's Mother had reported that her son's behaviour was 'out of control' a year before ... she had called the police to report her son missing stating that she was struggling to manage his behaviour and that he was returning home with unexplained amounts of money and would pack a bag and stay with friends. (Study 1, Case 1)

In seven cases young people physically assaulted their parents/carers following experiences of peer-on-peer abuse and/or during the period of the professional response to those experiences. Young people were particularly abusive to parents after they had been victimised by peers or partners and on occasions when parents sought to prevent them from spending time with abusive peers or partners.

For some young people, risks that they were exposed to at home (such as domestic abuse or parental alcoholism) undermined the ability of their parents to keep them safe. However, as in the example previously outlined it was often factors external to the family, such as the pull of peer influence, bullying in school, or victimisation on public transport, that informed a young person's behaviour - behaviour that was often at odds with parental expectations. Yet during professional intervention it was the capacity of parents, and not factors that undermined parental capacity, that was the subject of assessment and intervention.

In addition to illuminating the contextual interplay involved in the undermining of parental capacity to safeguard, the CCR methodology recognises the same potential capacity, or lack thereof, of all contexts in which young people spend their time. The review process specifically collects examples of attempts made by young people, professionals within schools, and individuals/companies who operate in public spaces, to intervene in the escalation towards an abusive incident. Cases reviewed in both studies, for example, 
evidenced the struggle faced by schools when they sought to disrupt escalating bullying and harassment prior to sexual assaults within (and associated to) educational establishments:

The school struggled to manage Greg's behaviour and the level disruption that it caused. Over the five years they used detentions, a step process towards other sanctions, removal from class, internal isolation and a fixed-term exclusion (Study 2, Case 4)

School moves Lacey to another part of the school to avoid contact with the boy ...Lacey is having 'problems' with another girl her class - school change Lacey timetable so that she is not in class with this girl. In the following month ...' In the New Year logged that Lacey has been threatened by someone from a different school... school assign a mentor to Lacey - the following week Lacey is recorded as making 'rude and offensive comments during mentoring time, threatening a member of staff' school gives a fixed term 3 day exclusion. (Study 1, Case 4)

Professionals, peers and members of the public did not have the capacity to safeguard young people from the significant harm that they encountered in public spaces. Peers attempted to intervene and disrupt abuse in nine cases but in all bar one were unable to do so. Professionals who struggled to safeguard young people in their local community relocated them out of unsafe spaces that were beyond their control.

As a result, the CCR methodology highlights both when parental capacity is undermined by extra-parental issues (and the extent to which this is recognised), and the capacity of all those who inform, or are informed by, the social contexts in which young people encounter significant harm. In doing so, the review process moves away from assessing the professional response to young people and their families and towards the professional influence over, and involvement with, the contexts associated to peer-on-peer abuse.

\section{Utilising child protection assessments and procedures}

Section (3) of the CCR template collects evidence on the interventions and the assessments used in response to a peer-on-peer abuse incident. This information is not collated to ascertain whether procedures were followed. Instead the template explicitly notes which contexts were considered in assessment and intervention, and the impact that the response had on all contexts featured in the case. The template can then be analysed to compare where risk was located (Sections 1 and 2) and where the response was focused (Section 3).

The use of CCRs has identified that even when professionals apply child protection procedures to safeguard young people from extra-familial risk, these responses have largely involved the assessment of, and intervention with, affected families. As such, risk within peer groups, schools and neighbourhoods associated to the incidents under review at best persisted (and were unaddressed), or at worse escalated. As alluded to in the two previous subsections, the risk of significant harm in extra-familial contexts was often responded to by assessing the capacity of parents to protect their children from peer-on-peer abuse. In the absence of contextual change, or parental capacity to influence contextual change, families were relocated or individual young people were taken into local authority care in the two studies. 
The CCR process draws out the limitations of safeguarding processes which fail to engage with contexts where peer-on-peer abuse actually escalates and in doing so raises questions about the sufficiency of wider safeguarding procedures and legislation, as well as their application in local areas. For those employing the CCR approach it is not enough to conclude that professionals followed all procedures correctly but that this was insufficient to safeguard a young person. If the response didn't engage with the locale of vulnerability then reviewers would conclude that professionals fell short of their duty safeguard young people. When making recommendations, reviewers would then be required to consider what would prevent that shortfall again. Does the solution lie in a different interpretation of existing legislative parameters or is legislative change required to safeguard young people from peer-on-peer abuse and other forms of extra-familial risk?

\section{Discussion: Implications for practice and policy}

Employing a CCR methodology illuminates three things about the nature of peer-on-peer abuse and current responses to it, which in turn has wider implications for review practices and child protection policy. Firstly CCRs illustrate the interplay that occurs between different extra-familial contexts which would otherwise be subsumed within one shared category of 'environmental factors'. Secondly, it provides space to explore the ways which parental capacity can be undermined by extra-familial risk while simultaneously raising questions about the capacity of peers and professionals to safeguard the young people with whom they interact. Finally, it identifies the extent to which safeguarding responses engage with the contexts in which risk actually occurs/escalates as opposed to where processes require engagement. :

In doing so it questions whether legislative, structural, or organisational reform is required to provide frameworks for safeguarding young people from extra-familial risk. In particular whether the following two 'concepts' of child protection - 'significant harm' and 'capacity to safeguard' - should be applied to extra-familial contexts as follows:

1. If a young person experiences 'significant harm' in a peer group, school or publicspace environment then should that environment be subject to assessment and intervention in the same way it would be if a home environment was the location of the abuse?

2. Can the notion of 'capacity to safeguard' be applied at organisational and social, as well as familial, levels? Rather than scrutinising whether services support parents to safeguard young people from extra-familial risk, CCRs suggest the examination of whether professionals, peers and families intervened with, and altered, the contexts of extra-familial harm.

To transfer these principles of child protection would likely require changes in guidance and protocol, potentially legislation, and cultural attitudes to extend the remit of safeguarding to public and social spaces - an approach arguably inconsistent with reactive, individual and proceduralised models of child protection (Parton, 2014). Conceptually if such a model were realised then the leaders of a school environment, a housing association, or a shopping centre could be assessed via child protection systems in ways currently reserved for parents/carers. If young people are being abused within the environment that these individuals are responsible for then it is their capacity to safeguard which would be subject to interrogation. 
Furthermore, if the nature of one of those environments was undermining the capacity of a parent, teacher or youth worker to safeguard a young person then this interplay would need to be both acknowledged in assessment and used to guide intervention. For example a young woman continues to run from home, despite boundaries put in place by her parents, so that she can go to the local park where a group of peers have groomed her into exploitative sexual acts. Intervening with that young woman's family is unlikely to disrupt the behaviour of that peer group or make the park they are in any safer - and is therefore also unlikely to address factors undermining parental capacity to safeguard her. The park, and the peer group who use it for the purposes of abuse, however, could be subjected to assessment and intervention.

While this discussion has arisen from CCRs in England, it is significant for all similar social work systems - i.e. those that use a 'child protection' as opposed to a 'family support' model (Connolly, 2016). At a time when case reviews are under new scrutiny in the UK and responses to adolescent safety are subject to international debate (DfE, 2016; United Nations, 2015) such conceptual questions, while challenging, are of critical importance. Although the CCR methodology highlights these challenges, the legislative parameters that support child protection work are some distance from applying this conceptual position.

At this stage $18 \mathrm{CCRs}$ have been conducted and a further two have been commissioned. While these will span 13 local authorities and four police forces in England, contextual scrutiny of safeguarding practices are largely in their infancy. Far more contextual analysis of responses to peer-on-peer abuse and other safeguarding issues is required. More importantly, while CCRs raise questions about the sufficiency of child protection processes, the conceptual shift in operations suggested by this discussion requires development. Were more contextual responses to parks, peers groups, bus routes and schools piloted in local areas, for example, then case review recommendations could reference such exemplars. In the absence of contextual practice models CCRs tend to recommend changes that are beyond traditional child protection parameters, guidance or protocol.

In applying a systemic lens to safeguarding practices, CCRs illuminate the limitations of operational responses to peer-on-peer abuse and the wider legislative and cultural contexts in which these sit. By explicitly recognising interplay between different extra-familial contexts and considering the role of professionals within these contexts, CCRs apply systemic theories in ways that are complimentary to, albeit distinct from, other systemic case review methodologies. Further dissemination and development of this model, and associated tools, will enable other case review authors to apply CCR principles. An increase in CCRs will in turn contribute to the promotion of a cultural shift for advancing the safeguarding of adolescents, particularly from extra-familial harm, in a range of child protection systems that are primarily focused on the family.

\section{Bibliography}

Barter, C., McCarry, M., Berridge, D. \& Evans, K., 2009. Partner Exploitation and Violence in Teenage Intimate Relationships, London: NSPCC.

BBC, 2015. School sex crime reports in UK top 5,500 in three years. [Online]

Available at: http://www.bbc.co.uk/news/education-34138287

[Accessed 03. 12. 2015]. 
Beckett, H. et al., 2013. It's wrong but you get used to it: A qualitative study of gangassociated sexual violence towards, and sexual exploitation of, young people in England, London: Office of the Children's Commissioner.

Bourdieu, P., 1992. An invitation to reflexive sociology. Chicago: University of Chicago Press.

Brandon, M. et al., 2012. New learning from serious case reviews: a two year report for 2009-2011, London: Department for Education .

Connolly, M., 2016. Reforming child protection: Insights from Australaisia. [Online] Available at: http://www.kcl.ac.uk/sspp/policyinstitute/scwru/events/socialworkseminars/2016/Marie-Connolly-Presentation-27-6-16.pdf [Accessed 30. 06. 2016].

Corr, M. L., Gadd, D., Butler, I. \& Fox, C. L., 2012. From Boys to Men: Phase Two Findings, Manchester: University of Manchester School of Law.

DfE, 2016. Wood Report: Review of the role and functions of Local Safegaurding Children's Boards, London: Crown Copyright .

Firmin, C. 2015. Peer on Peer Abuse: Safeguarding Implications of Contextualising Abuse between Young People within Social Fields. Professional Doctorate Thesis. Luton: University of Bedfordshire.

Firmin, C. with Curtis, G., Fritz, D., Olatain, P., Latchford, L., Lloyd, J. and Larasi, I. 2016. Towards a Contextual Response to Peer-on-Peer Abuse. Luton: University of Bedfordshire.

Firmin, C. 2017. Contextual Risk, Individualised Responses: An Assessment of Safeguarding Responses to Nine Cases of Peer-on-Peer Abuse. Child Abuse Review DOI 10.1002/car.2449

Finkelhor, D., Ormrod, R. \& Chaffin, M., 2009. Juveniles Who Commit Sex Offences Against Minors. s.I.:Office of Juvenile Justice and Delinquency Prevention, US Department of Justice.

Fox, C. L., Corr, M. L., Gadd, D. \& Butler, I., 2012. From Boys to Men: Phase One Findings, Manchester: University of Manchester School of Law.

Hackett, S., 2011. Children and Young People with Hamrful Sexual Behaviours. In: Children Behaving Badly? Peer Violence Between Children and Young People. West Sussex: John Wiley \& Sons Ltd., pp. 121-136.

Letourneau, E. \& Borduin, C., 2008. The Effective Treatment of Juveniles Who Sexually Offend: An ethical imperative. Ethics and Behavior, 18(2-3), pp. 286-306.

Losel, F. \& Bender, D., 2006. Risk factors for serious and violence antisocial behaviour in children and youth. In: Children Who Commit Acts of Serious Interpersonal Violence: Messages for Best Practice. London: Jessica Kingsley, pp. 42-73.

Munro, E., 2011. The Munro Review of Child Protection: Final Report, A child-centred system, London: The Stationary Office. 
Parton, N., 2014. The Politics of Child Protection. Basingstoke: Palgrave.

Powell, A., 2010. Sex, Power and Consent: Youth Culture and the Unwritten Rules.

Cambridge: Cambridge University Press.

Radford, L. et al., 2011. Child abuse and neglect in the UK today, London: NSPCC.

Ringrose, J., Gill, R., Livingstone, S. \& Harvey, L., 2011. A Qualitative Study of Children, Young People and 'Sexting', London: NSPCC.

SCIE, 2012. Learning together to safeguard children: a 'systems'. [Online] Available at: http://www.scie.org.uk/publications/ataglance/ataglance01.pdf [Accessed 12. 12. 2015].

Squires, P. \& Goldsmith, C., 2011. Bullets, Blades and Mean Streets: Youth Violence and Criminal Justice Failure. In: Children Behaving Badly? Peer Violence Between Children and Young People. West Sussex: John Wiley and Sons Ltd, pp. 199 - 216.

United Nations, 2015. CRC call for submissions on its General Comment on the Rights of Adolescents. [Online]

Available at: http://www.ohchr.org/EN/HRBodies/CRC/Pages/CallRightsofAdolescents.aspx [Accessed 01. 04. 2016].

Walker, J. and Firmin, C. 2017, forthcoming. Contextual challenges of peer-on-peer abuselessons from serious case reviews 